\title{
Quand Georges Lefebvre, Alexandre de Saint-Léger et Paul Thomas passaient le diplôme d'Études supérieures d'histoire et de géographie
}

Philippe Marchand

\section{(2) OpenEdition \\ Journals \\ Édition électronique \\ URL : https://journals.openedition.org/ahrf/13570 \\ DOI : 10.4000/ahrf.13570 \\ ISSN : 1952-403X \\ Éditeur : \\ Armand Colin, Société des études robespierristes}

Édition imprimée

Date de publication : 1 décembre 2015

Pagination : 157-170

ISBN : 9782200930028

ISSN : 0003-4436

Référence électronique

Philippe Marchand, «Quand Georges Lefebvre, Alexandre de Saint-Léger et Paul Thomas passaient le diplôme d'Études supérieures d'histoire et de géographie », Annales historiques de la Révolution française [En ligne], 382 | octobre-décembre 2015, mis en ligne le 01 décembre 2018, consulté le 22 avril 2022. URL : http://journals.openedition.org/ahrf/13570 ; DOI : https://doi.org/10.4000/ahrf. 13570 
SOURCES

\title{
QUAND GEORGES LEFEBVRE, ALEXANDRE DE SAINT-LÉGER ET PAUL THOMAS PASSAIENT LE DIPLÔME D'ÉTUDES SUPÉRIEURES D'HISTOIRE ET DE GÉOGRAPHIE
}

\author{
Philippe MARCHAND
}

Dans l'introduction qu'il écrit en 1963 pour les Études sur la Révolution française de Georges Lefebvre, Albert Soboul donne quelques brèves indications sur son parcours scolaire de l'école primaire au baccalauréat ${ }^{1}$. Il puise son information dans les indications fournies par Georges Lefebvre lui-même ${ }^{2}$. En revanche rien n'est dit de ses études supérieures entreprises à la Faculté des lettres de Lille, études sur lesquelles l'auteur des Paysans du Nord est resté silencieux. Nos recherches sur l'enseignement de l'histoire à la Faculté des lettres de Lille dans les années où Georges Lefebvre y est étudiant nous ont permis de retracer son parcours et d'avoir un aperçu de ses débuts dans le métier d'historien. Ces débuts, on peut les suivre dans le rapport que Charles Petit-Dutaillis donne de la première session d'examens organisée à Lille pour l'obtention du diplôme d'études supérieures d'histoire et de géographie les 26, 27 et 28 juin $1897^{3}$. Y participent trois candidats : Georges Lefebvre, Alexandre de Saint-Léger et Paul Thomas.

(1) Georges LEFEBVRE, Études sur la Révolution française Introduction par Albert SoBOUL, Paris, Presses Universitaires de France, Deuxième édition revue, 1963, p. 1-22.

(2) Idem, «Pro Domo », AHRF, 1946, n 105, p. 188-190.

(3) AD Nord 2 T 922, Rapport présenté par M. Petit-Dutaillis chargé d'un cours d'histoire du moyen âge sur la session d'examens de Juin 1897 pour l'obtention du diplôme d'études supérieures d'histoire et de géographie. 


\section{Le diplôme d'études supérieures d'histoire et de géographie}

En préalable à la présentation du rapport de Charles Petit-Dutaillis, il faut dire quelques mots de cet examen créé par l'Arrêté relatif au concours de l'agrégation d'histoire et de géographie du 28 juillet $1894^{4}$. Son objectif est de remédier à l'insuffisance de la formation scientifique des candidats à l'agrégation d'histoire et de géographie. Décerné par les facultés des lettres, l'École normale, l'École des Chartes et l'École des Hautes études, obligatoire pour s'inscrire à l'agrégation, le diplôme d'études supérieures qui vient s'ajouter aux trois grades traditionnels (baccalauréat, licence et doctorat) comprend plusieurs épreuves : un examen et discussion d'un mémoire d'histoire ou de géographie, dont le sujet a été choisi par le candidat et agréé par les professeurs ou maîtres de conférences d'histoire et de géographie ; la discussion d'une question d'histoire ou de géographie indiquée au candidat trois mois à l'avance par les professeurs et maîtres de conférences, la question étant choisie dans des périodes de l'histoire ou des parties de la géographie autres que celle à laquelle appartient le sujet du mémoire ; une explication critique d'un texte historique ou d'un texte géographique choisi par le candidat et agréé par les professeurs et ou maîtres de conférences ; une épreuve tirée au choix du candidat, soit des études auxiliaires de l'histoire (archéologie, épigraphie, paléographie, diplomatique, bibliographie), soit de la géographie générale.

\section{Les candidats}

Né à Lille le 6 août 1874, fils d'un comptable, Georges Lefebvre fait ses études primaires dans une école primaire publique de la ville. Remarqué par ses maîtres, il obtient une bourse communale qui lui permet d'entrer au lycée de Lille, mais dans l'enseignement secondaire spécial, c'est-à-dire dans cette filière sans latin ni grec créée par Victor Duruy. «On y apprenait », écrit Georges Lefebvre, « deux langues vivantes, beaucoup de mathématiques et de sciences de la nature, aussi l'économie politique et du droit». Cet enseignement, souligne-t-il, lui a ouvert «l'esprit aux réalités économiques et sociales $»^{5}$. Reçu au baccalauréat de l'enseignement

(4) Sur cet examen, voir Philippe MARCHAND, L'histoire et la géographie dans l'enseignement secondaire. Textes officiels Tome I : 1795-1914, Paris, INRP, 2000, p.75-84, p. 621-631 ; André CHERVEL, Histoire de l'agrégation. Contribution à l'histoire de la culture scolaire, Paris, Éditions Kymé, 1993, p. 174-182.

(5) Georges LefEBVRE, « Pro Domo » ... art. cit. 
secondaire spécial en août $1892^{6}$, il entre en classe de philosophie et est reçu à la seconde partie du baccalauréat série philosophie en juillet 1893 , mention bien ${ }^{7}$. Toujours titulaire d'une bourse, il entre en octobre à la Faculté des lettres de Lille pour y préparer une licence ès lettres mention histoire et, là, il doit se mettre à l'étude du latin et du $\operatorname{grec}^{8}$. Ses premiers résultats, 7 sur 20 à la composition latine du $1^{\text {er }}$ trimestre, 4 à celle du $3^{\mathrm{e}}$ trimestre, ne le découragent pas. Au terme de cette année 1893-1894, il passe le concours pour l'obtention d'une bourse de licence' ${ }^{9}$. Avec un total de 90 points sur 120, il est classé second sur seize candidats. Et dans son rapport, le doyen Angellier écrit :

« Je remarque enfin avec grand plaisir que des élèves suivant les cours à leurs frais ont bien réussi : Lesage 1er, Lefebvre 2e. Lefebvre n'a que 7 en latin mais il nous était arrivé à l'état nul sortant de l'enseignement spécial, il a fait des progrès sensibles et constants ${ }^{10}$.

Les années 1895-1896 et 1896-1897 sont consacrées à la licence. Ses notes en latin n'atteignent jamais la moyenne ${ }^{11}$. Mais ses résultats en français et, surtout, en histoire et en géographie, lui permettent de combler son handicap ${ }^{12}$. À la rentrée de l'année 1896-1897, il obtient une bourse d'agrégation d'un montant de 250 francs par trimestre. Il lui faut avant de passer le concours obtenir le diplôme d'études supérieures qu'il prépare tout en suivant les cours d'agrégation.

Né à Dunkerque le 3 avril 1866, Alexandre de Saint-Léger a fait ses études secondaires au collège communal de Dunkerque ${ }^{13}$. Bachelier

(6) AD Nord 2 T 1368, Registre des brevets de capacité et diplômes de l'enseignement secondaire spécial, puis baccalauréat à partir de juillet 1883. Le registre ne mentionne que les noms des lauréats.

(7) Ibidem, 2 T 2848, Procès-verbaux de l'examen pour l'obtention du baccalauréat, juillet 1893. En histoire, Georges Lefebvre est interrogé sur Les places assiégées en 1870 ; en géographie, sur Belfort. Il est noté 18/20.

(8) La licence ès lettres comprend des épreuves communes et des épreuves spéciales (lettres, philosophie, histoire). Dans les épreuves communes figurent à l'écrit une composition en latin à l'écrit, l'explication d'un auteur grec et d'un auteur latin à l'oral.

(9) Les bourses de licence et les bourses d'agrégation sont créées par les décrets de 1877 et 1880 .

(10) AD Nord 2 T 822, Rapport du doyen de la Faculté des lettres sur les examens, juillet 1894.

(11) 6/20 aux compositions du second et du troisième trimestre de l'année 1894-1895, 8/20 aux compositions du premier et du second trimestre de l'année 1895-1896.

(12) Les notes en histoire et en géographie ne sont jamais inférieures à 14/20. Il en va de même en français.

(13) Édouard PERroy, « Notice nécrologique d'Alexandre de Saint-Léger (1866-1944) », Revue du Nord, $\mathrm{n}^{\circ} 105,1944$, p. 165-170 
en $1886^{14}$, il occupe un poste de maitre d'études au collège de Dunkerque pendant l'année scolaire 1886-1887. Il quitte Dunkerque pour Lille où, tout en remplissant la fonction de maître auxiliaire au lycée, il s'inscrit à la Faculté des lettres. Élève de Paul Fabre (médiéviste) et surtout de Jules Flammermont, spécialiste du XVIII ${ }^{\mathrm{e}}$ siècle, qui décidera de son orientation future, il obtient la licence ès lettres mention histoire en 1890. Boursier d'agrégation, il est nommé professeur d'histoire à l'École supérieure de commerce de Lille en octobre 1893.

Né le 18 avril 1874 au Puy (Haute-Loire), Jean-Paul Thomas est le fils d'Émile Thomas, professeur de littérature latine à la Faculté des lettres de Lille. Il fait ses études secondaires au lycée de Lille. Bachelier en $1892^{15}$, il s'inscrit en qualité d'étudiant libre à la Faculté des lettres où il obtient la licence ès lettres mention histoire.

\section{L'examen}

Le jury est composé de Charles Petit-Dutaillis, chargé de cours d'histoire médiévale, de Jules Flammermont, professeur d'histoire moderne, de Gustave Fougères, chargé d'un cours complémentaire d'archéologie et d'histoire de l'art, d'Édouard Ardaillon, chargé de cours de géographie, et d'Ernest Haumant, professeur de langue et littérature russes ${ }^{16}$.

Le rapport de Charles Petit-Dutaillis ne donne aucune information sur la durée de soutenance des examens des candidats. Une indication nous est fournie dans les rapports hebdomadaires de la faculté pour l'année 1895-1896 qui précise que l'examen du diplôme d'études supérieures de Louis Germain a duré de 14 heures 30 à 17 heures ${ }^{17}$. On peut penser qu'il en est allé de même lors de cette session de 1897 qui s'étend sur trois jours : 24,25 et 26 juin.

(14) AD Nord 2 T 2829, $1^{\text {re }}$ partie, juillet $1885 ; 2$ T 2832, $2^{\mathrm{e}}$ partie, novembre 1886.

(15) Ibidem, 2 T 2841, $1^{\text {re }}$ partie, juillet 1891 , mention bien ; $2 \mathrm{~T} 2845,2^{\mathrm{e}}$ partie, juillet 1892 , mention passable.

(16) Ibid., VM 165 074, Registre des diplômes d'études supérieures soutenus. Pour plus de détails sur ces différents enseignants, $c f$. Jean-François CONDETTE, Les lettrés de la République Les enseignants de la faculté des lettres de Douai puis Lille sous la Troisième République (1870-1940) Dictionnaire biographique, Lille, IRHiS-CEGES-Université Lille 3, Collection Histoire de l'Europe du Nord-Ouest, 2006, p. 143, p. 169-171 et p. 204. Sur Ernest Haumant, $c f$. André LIRONDELLE, « Ernest Haumant Nécrologie », Revue des études slaves, 1944, vol. 21-1-4, p. 174-177.

(17) AD Nord 2 T 898, Faculté des lettres de Lille, Rapports hebdomadaires 1888-1900. 


\section{Résultats et commentaires du jury}

Au terme de ces trois journées, les trois candidats sont déclarés dignes du diplôme d'études supérieures, Alexandre de Saint-Léger avec la mention bien, Paul Thomas la mention assez bien, et Georges Lefebvre la mention passable. Le rapport sur cette session de juin de 1897 suggère plusieurs remarques. En premier lieu, on constate que deux des trois candidats, Alexandre de Saint-Léger et Paul Thomas, choisissent un sujet d'histoire locale nécessitant des dépouillements d'archives. Georges Lefebvre, sans doute sur les conseils de Flammermont, choisit un sujet qu' on peut ranger dans le domaine de l'histoire des idées politiques.

En second lieu, si le jury se félicite de la création de ce nouvel examen, il souhaite que soit revue sa relation avec le concours d'agrégation et demande un examen des coefficients attribués à chaque épreuve. Et surtout il critique les conditions dans lesquelles les candidats lillois doivent s'y préparer. À propos de la seconde épreuve - la discussion d'une question d'histoire indiquée trois mois à l'avance - il réclame la création d'un enseignement d'histoire ancienne confié à un spécialiste rompu à l'étude des textes. Ce vœu est exaucé en 1898 avec la nomination de Pierre Jouguet, chargé d'un enseignement d'histoire des institutions grecques et romaines, d'histoire ancienne et de papyrologie. Il souligne aussi une autre difficulté que rencontrent les candidats : l'absence d'ouvrages d'histoire moderne dans la bibliothèque et la lenteur avec laquelle les ouvrages demandés arrivent dans ce qui n'est pas encore l'Institut d'histoire. Tenant compte de cette situation, le jury attribue la même note aux trois candidats pour cette épreuve.

On notera aussi à propos de l'épreuve de géographie le plaidoyer d'Ardaillon pour une géographie qui n'oublie pas l'homme. Cette remarque renvoie au processus de transformation de la science géographique dans cette dernière décennie du XIX ${ }^{\mathrm{e}}$ siècle $^{18}$.

Enfin dernière remarque : si Georges Lefebvre n'obtient que la mention passable, sa prestation sur le Modus tenendi Parliamentum in Anglia ${ }^{19}$, notée $8 / 10$, lui vaudra d'être sollicité quelques années plus tard

(18) Paul VIDAL de la BLACHE, «Le principe de la géographie générale », Annales de géographie, 1896, 5, 20, p. 129-142.

(19) Le Modus Tenendi Parliamentum est un texte anglais ancien dont la date exacte de rédaction a fait l'objet de nombreuses discussions. Il traite de l'organisation et du fonctionnement du Parlement. La première édition savante est due à Thomas D. Hardy en 1846 (Thomas D. HARDY, The Parliament in England, Londres, 1846) avec le texte original en latin et sa traduction. Outre cette édition copieusement annotée, on peut penser que Georges Lefebvre a eu accès à l'article de Charles 
par Charles Petit-Dutaillis pour écrire une étude originale relative au Parlement anglais aux $\mathrm{XIII}^{\mathrm{e}}$ et $\mathrm{XIV}^{\mathrm{e}}$ siècles, insérée dans la traduction française de la Constitutionnal History of England de William Stubbs, puis traduite en anglais ${ }^{20}$.

\section{Le devenir des trois lauréats}

Alexandre de Saint-Léger, écoutant les conseils de son maître Flammermont, entreprend le dépouillement des archives anglaises et des archives de la Marine. Il obtient le doctorat ès lettres en 1900. Sa thèse principale, La Flandre maritime et Dunkerque sous la domination française (1659-1789) ${ }^{21}$, est une version enrichie de son mémoire de diplôme. Nommé chargé de cours à la Faculté des lettres de Lille, il y commence une longue carrière qui le voit se consacrer à la promotion de l'histoire locale et régionale.

Titulaire du diplôme d'études supérieures, Georges Lefebvre obtient le renouvellement de sa bourse d'agrégation pour l'année 1897-1898. Les notes qu'il obtient aux épreuves organisées à la faculté dans le cadre de sa préparation sont toujours largement supérieures à la moyenne : 16, 17, 18. Sa note la plus faible lui est attribuée pour une leçon d'oral : 12/20. Et il faut noter qu'il semble avoir comblé son retard en latin avec un 16/20 à une composition de version ${ }^{22}$. N'étant pas reçu au concours, il doit accepter un poste de professeur d'histoire et de géographie au collège de Boulogne. Il s'inscrit aux cours du jeudi organisés à la faculté pour les professeurs licenciés voulant préparer l'agrégation. En raison de l'éloignement, il ne peut y être assidu, ce qui ne l'empêche pas d'obtenir un 16/20 aux devoirs d'histoire et de géographie proposés dans le cadre de cette préparation. Au terme de l'année scolaire 1898-1899, il est reçu au concours $8^{\mathrm{e}}$ sur 11 admis $^{23}$. Commence alors pour l'auteur des Paysans du Nord une longue carrière dans l'enseignement secondaire (vingt-cinq années qui le voient passer par les lycées de Cherbourg, de Tourcoing, de Lille, puis par les

BÉMONT, « La date de composition du Modus Tenendi Parliamentum », publié dans les Mélanges Julien Havet en 1895 (p. 465-480).

(20) Charles PetiT-DUTAILlis et Georges LeFEBVRE, Studies and notes supplementary to Stubb's Constitutionnal History, Manchester, 1929. Sur cette collaboration, cf. Albert SoBOUL, Introduction... op. cit., p. 2-3, note 3.

(21) Alexandre de SAINT-LÉGER, La Flandre maritime et Dunkerque sous la domination française (1659-1789), thèse de doctorat, Paris-Lille, Tallandier, 1900.

(22) AD Nord 2 T 823, Notes obtenues par les boursiers d'agrégation en 1897/1898.

(23) André CHERVEL, Les lauréats des concours d'agrégation de l'enseignement secondaire 1821-1950, Paris, INRP, 1993, p. 68. On notera que les autres reçus de cette promotion ne sont guère passés à la postérité. 
grands lycées parisiens) tout en menant des recherches qui devaient en faire le maître incontesté de l'historiographie révolutionnaire.

Paul Thomas, le moins connu des trois, fait ses débuts dans l'enseignement secondaire au collège de Commercy en 1903. Professeur au lycée de Châteauroux en 1904, il est reçu à l'agrégation d'histoire et de géographie en 1910. Il revient dans le Nord et enseigne au lycée de Tourcoing (1911-1919), puis au lycée Faidherbe de Lille (1919-1936). S'il n'a jamais soutenu de thèse, Paul Thomas ne délaisse pas la recherche historique. Il est l'auteur de plusieurs articles sur la période qui avait été celle de son DES et de Textes historiques sur Lille et le nord de la France avant $1789^{24}$. Il a laissé un ensemble impressionnant de notes historiques accumulées lors de ses recherches dans les Archives du Nord.

Pour conclure la présentation de ce document, on peut récapituler ses principaux apports. Il fournit d'abord de précieuses indications sur le contenu d'un examen qui en est encore à ses débuts. Il nous renseigne sur les difficultés rencontrées dans sa préparation par les étudiants dans une faculté de province : insuffisance des ressources bibliographiques, absence d'enseignants spécialisés. Enfin, il montre les débuts de jeunes historiens dont deux devaient accéder à une grande notoriété, l'un, Alexandre de Saint-Léger, sur le plan régional, l'autre, Georges Lefebvre au niveau international.

(24) Paul Thomas, Textes historiques sur Lille et le Nord de la France avant 1789 (imprimés et inédits), Paris-Lille, Bibliothèque de la Société d'histoire du Droit et des Pays flamands, picards et wallons, 1936, 2 vol. 


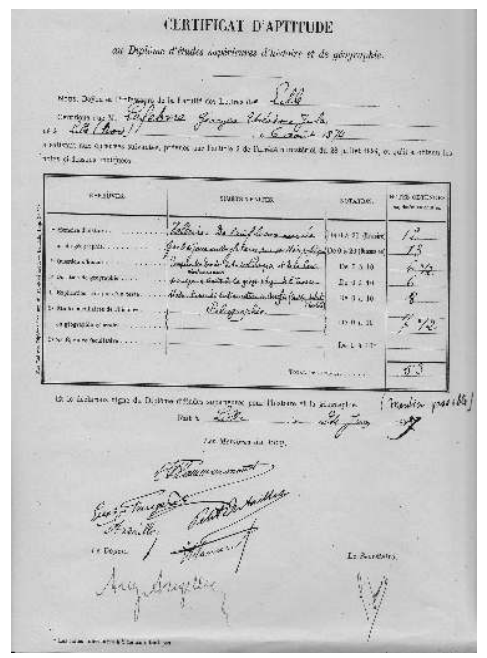

Rapport présenté par M. Petit-Dutaillis chargé du cours d'histoire du moyen âge sur la session d'examens de juin 1897 pour l'obtention du diplôme d'études supérieures d'histoire et de géographie

Cette année la session des examens pour l'obtention du diplôme d'études supérieures d'histoire et de géographie à la Faculté des Lettres de Lille a présenté un intérêt considérable dû au nombre relativement important des candidats, à la valeur de leurs travaux et à leur qualité d'élèves ou d'anciens élèves de la Faculté. Cet examen qui forme maintenant le premier degré de l'agrégation d'histoire et de géographie comporte quatre sortes d'épreuves : $1^{\circ}$ Remise et discussion d'une thèse manuscrite faite à loisir sur un sujet choisi longtemps d'avance, d'accord avec les membres du jury ; $2^{\circ}$ Discussion d'une question d'histoire et d'une question de géographie indiquées au candidat trois mois d'avance ; $3^{\circ}$ Explication critique d'un texte historique ou d'un texte géographique choisi par le candidat et agréé par les professeurs ; $4^{\circ}$ Une épreuve tirée au choix du candidat soit des études auxiliaires à l'histoire (archéologie, épigraphie, paléographie, diplomatique, bibliographie) soit de la géographie générale.

Sur trois thèses, deux étaient relatives à l'histoire du Nord de la France. M. de Saint-Léger, ancien élève de la Faculté, professeur à l'École supérieure de commerce de Lille, avait choisi pour objet de son travail écrit «Dunkerque et la Flandre maritime sous la domination française (1662-1789) ». Ce petit pays, correspondant aux arrondissements actuels de Dunkerque et d'Hazebrouck, a eu dans le dernier siècle du régime monarchique une situation particulière tout à fait intéressante à examiner 
dans le détail et qui montre au vif quelle était dans l'ancienne France la complexité de l'organisme administratif et à la fois la puissance et la force de résistance de la vie locale. Quand cette contrée faisait partie des Pays-Bas Espagnols, elle envoyait aux États de Flandre les députés de ses villes chefs-collège ${ }^{25}$ et contribuait par leurs suffrages à voter le subside annuel payé par la province. Bien qu'après l'achat de Dunkerque en 1662 les privilèges de la Flandre maritime eussent été confirmés, Louis XIV ne lui accorda pas dans la réalité le consentement des impôts et sans être pays d'élection ni pays d'états, elle fut comme les pays d'élection et les pays d'états écrasée jusqu'à la fin de l'ancien régime par une pesante fiscalité. Cependant, elle ne fut pas absolument privée d'autonomie financière ; elle continua à voter des fonds pour ses propres dépenses, eut le droit de répartir les impôts et la faculté même de les lever à son gré moyennant le payement d'un abonnement. De même si le souverain lui envoya un intendant, un bailli, un maître des eaux et forêts etc... Elle garda la même administration locale, ses châtellenies, ses « Magistrats », son assemblée du « Département ». Sans méconnaître les grands bienfaits apportés par la domination française, les progrès de l'agriculture et surtout du commerce maritime les habitants gardaient l'esprit d'initiative et de personnalité ; dans les cahiers de 1789, ils protestent contre le despotisme de l'intendant et du subdélégué général et réclament l'institution d'états provinciaux.

Tels sont les principaux faits que $\mathrm{M}$. de $\mathrm{S}^{\mathrm{t}}$ Léger a révélés ou mis en plus vive lumière ; l'étendue de ses investigations, la fermeté de sa méthode critique, la netteté de son plan et de son style témoignent d'un esprit réfléchi et mûr ; l'exposé oral qu'il a fait de ses découvertes essentielles aussi bien que son travail écrit lui ont valu les félicitations unanimes du jury qui lui a décerné une note très élevée, 18 sur 20, pour son mémoire, et la note 15 pour la soutenance.

Qu'il complète ses recherches surtout en interrogeant les archives anglaises et les archives du Ministère de la marine, qu'il élargisse et aère pour ainsi dire une rédaction un peu trop serrée, qu'il donne selon la méthode actuelle les preuves de ses allégations et il pourra soumettre à la Faculté une excellente thèse de doctorat ès lettres.

M. Paul Thomas, étudiant à la Faculté de Lille, s'est proposé de décrire d'après les archives de la région «Le gouvernement du duc de Bourgogne Jean sans Peur dans le comté de Flandre ». La richesse même

(25) Les chefs-collèges sont des assemblées représentant à la fois les chefs-lieux (Bailleul, Bergues, Bourbourg et Cassel) et l'ensemble des châtellenies. Ils s'occupent de la levée des impôts, de l'entretien des voies de communication. 
de ses archives l'a forcé à arrêter ses recherches et à ne parler même que d'une des parties des notes qu'il avait recueillies à Lille ou en Belgique. Il s'est décidé, un peu tardivement peut-être, à étudier deux parties du sujet $1^{\circ}$ Les registres des chartes de Jean sans Peur $2^{\circ}$ La campagne de $1414^{26}$. L'étude diplomatique des registres, faite avec une grande minutie et une patience de recherches qui révèlent un goût véritable pour l'érudition, a été accompagnée d'un catalogue des chartes portant la suscription de Jean sans Peur. Ce catalogue, très volumineux a été fait trop rapidement et les analyses ne sont pas toujours exactes. Le récit de la campagne de 1414 vaut mieux. Il s'agit d'un épisode de la lutte des Armagnacs et des Bourguignons, de la guerre civile qui a précédé immédiatement le désastre d'Azincourt. Il était fort intéressant de savoir par le menu et surtout au moyen des renseignements irrécusables que fournissent les actes et les comptes financiers quelle était alors la situation de Jean sans Peur, quelles étaient les intrigues secrètes, pourquoi il a été obligé de signer la paix d' $\operatorname{Arras}^{27}$ et pourquoi il s'est trouvé réduit à l'effacement et à la neutralité au moment de l'invasion anglaise.

M. Paul Thomas a montré que Jean sans Peur, obligé par sa propre politique à mettre sur pied une armée d'un entretien extrêmement coûteux, s'était vu refuser tout subside par les Flamands qui voulaient profiter de l'occasion pour lui arracher la concession d'une sorte de gouvernement autonome. Acculé au dernier degré de la détresse financière, le duc s'humilia devant ses ennemis et signa le traité d'Arras, non cependant sans obtenir de la faiblesse du dauphin des articles secrets dont M. Thomas a découvert le texte et qui jettent une grande lumière sur les événements postérieurs. Le seul défaut grave de cette étude est qu'elle manque d'ampleur. Le sujet n'a pas été examiné ni présenté dans son ensemble. M. Thomas a fait porter trop exclusivement ses recherches sur les rapports de Jean sans Peur avec les Flamands ; certains autres côtés de la question, tout aussi intéressants et tout aussi obscurs, ont été négligés.

Malgré ces lacunes, bien excusables d'ailleurs, la thèse de ce candidat a paru mériter la note 14 , de même que sa soutenance.

M. Lefebvre avait choisi un sujet en apparence plus facile «L'Influence du séjour de Voltaire en Angleterre (1726-1729) sur ses idées politiques ». Pour traiter un pareil sujet, il fallait étudier à fond la vie et les idées de Voltaire avant ce voyage qu'il entreprit dans un état

(26) Épisode de la guerre civile opposant Armagnacs et Bourguignons.

(27) Conclue le 4 septembre 1414 entre les représentants de Jean sans Peur et Louis de Guyenne, représentant son père Charles VI. 
d'âme si spécial ${ }^{28}$ et ensuite les ouvrages postérieurs et surtout ceux qui ont été directement inspirés par son voyage Outre-Manche ; il aurait fallu par exemple examiner minutieusement Les Lettres sur les Anglais ${ }^{29}$ et l'édition de la Henriade donnée en $1728^{30}$, la première avouée par l'auteur. Il est remarquable que Voltaire depuis son retour n'ait pas cessé de s'occuper de politique. M. Lefebvre a fait certainement des lectures très étendues et, autre mérite, s'est attaché à traiter le sujet historiquement en étudiant la situation de l'Angleterre à l'époque où Voltaire y habita ; mais faute sans doute d'avoir réservé assez de temps pour mettre en œuvre les matériaux amassés, il a cru pouvoir conclure que le philosophe n'avait fait aucun emprunt spécial aux idées politiques des Anglais, appréciation qui a semblé inacceptable au jury. La thèse a été cotée 12, l'exposé oral préparé avec un soin peut-être excessif, et parfois obscur à force d'être abstrait, a obtenu la note 12 .

D'une façon générale les thèses ${ }^{31}$ données trois mois d'avance ont été moins bonnes. Les candidats visiblement n'y ont pas consacré assez de temps. C'est ce qui est apparu soit dans les résumés qu'ils ont remis quelques jours avant l'examen, soit pendant la discussion orale. M. de $S^{t}$ Léger a eu à préparer une question sur les institutions de la Crète. L'intérêt du sujet était dans le rapprochement fait par les auteurs anciens eux-mêmes entre les institutions de la Crète et celles de Sparte, en particulier dans l'identification établie par eux entre les Cosmes crétois ${ }^{32}$ et les Ephores de Sparte ${ }^{33}$. Il fallait ensuite montrer le caractère de la législation crétoise d'après l'inscription de Gortyne ${ }^{34}$, enfin rechercher les origines du Kö̈non ${ }^{35}$. M. de $\mathrm{S}^{\mathrm{t}}$ Léger

(28) Allusion au séjour de Voltaire à la Bastille où il est enfermé en 1726 pour la seconde fois pendant cinq mois pour avoir provoqué en duel le chevalier de Rohan. Sa libération lui est accordée à la condition qu'il ne cherche plus à provoquer Rohan et qu'il réside en Angleterre, ce qu'il fait pendant trois ans.

(29) Publiées en 1734, ces vingt-cinq lettres abordent des sujets très variés : religion, sciences, art, politique, philosophie.

(30) Commencée en 1718, la Henriade, poème à la gloire d'Henri IV, fait l'apologie de la tolérance. Elle est publiée en 1728 pendant le séjour de Voltaire en Angleterre et grâce à une souscription qui connaît un grand succès auprès des Anglais.

(31) Il s'agit ici de la seconde épreuve : discussion d'une question d'histoire et d'une question de géographie.

(32) Les Cosmoi constituaient dans les principales cités crétoises des collèges d'une dizaine de membres, élus par les grandes familles et disposant de pouvoirs civils et militaires considérables (ARISTOTE, La Politique, Livre VII chapitre VII).

(33) Collège de cinq magistrats constituant le véritable gouvernement de Sparte.

(34) Datée de la première moitié du $\mathrm{V}^{\mathrm{e}}$ siècle av. J.-C., cette inscription, découverte à Gortyne, atteste l'existence dans les cités crétoises d'une importante population dépendante intermédiaire entre la condition d'homme libre et celle d'esclave portant des noms divers (oikees à Gortyne) selon les cités (Claude Mossé, Dictionnaire de la civilisation grecque, Bruxelles, Complexe, 1998).

(35) Sous le terme de Koinon, on désigne une forme de coordination et d'unité politiques dans le cadre de ligues régionales de cités et de tribus autonomes. Ces communautés d'États étaient dotées 
a juxtaposé avec diligence les faits, sans en tirer les discussions et les conclusions qui faisaient l'intérêt du sujet ; il s'est arrêté en chemin.

M. Lefebvre devait comparer les données de la lex Pompeia ${ }^{36}$ et de la lex Concilii Narbonensis ${ }^{37}$. Sur la demande du jury, il a expliqué la différence qui séparait les leges datae de la République, destinées simplement à organiser les conquêtes, des lois impériales assurant aux provinces le maintien ou la création d'un système d'assemblées fédérales. Il a commis une erreur grave, mais fort commune, sur le culte des empereurs. Il a ensuite expliqué et commenté le fragment conservé de la Lex Narbonensis, l'explication a été bonne, le commentaire pauvre.

L'une et l'autre de ces épreuves portant sur les institutions grecques et romaines ont prouvé que nos étudiants n'ont pas les qualités nécessaires pour étudier à fond l'histoire de l'antiquité où l'interprétation, faute de textes nombreux, doit tenir une grande place. Il serait extrêmement désirable que dans une Université aussi importante que la nôtre, il y eût des cours et des conférences d'histoire ancienne et que cet enseignement, si utile pour la formation des esprits, au lieu d'être dû à la bonne volonté des professeurs qui n'en ont pas officiellement le soin et qui sont chargés de tout autres besognes, fût confié à un spécialiste.

M. Thomas avait à examiner la question de l'État autrichien en 1809. Il a lu, mais trop vite et trop tard, les ouvrages allemands relatifs à l'histoire de l'Autriche au temps de Napoléon; il n'a pas su distinguer le véritable intérêt de la question, ni montrer l'agitation sourde des nationalités, dont Napoléon se conformant d'ailleurs aux traditions de l'ancien régime, espéra profiter pour démembrer les états des Habsbourg jusqu'au jour où il se résigna à $« 1$ 'alliance intime $»^{38}$. Une circonstance indépendante de la volonté du candidat comme de l'action de ses professeurs, excuse la médiocrité de la préparation : la pauvreté de notre bibliothèque en livres

d'un pouvoir central fort. Les plus connues sont le koinon des Thessaliens, le koinon des Molosses d'Épire (Paschalis KITROMILIDES, « La Grèce un exemple historique de l'organisation confédérale des États » dans Les concepts contemporains de confédération, Strasbourg, Éd. du Conseil de l'Europe, Collection Sciences et techniques de la démocratie, $\mathrm{n}^{\circ} 11,1995, \mathrm{p} .10-16$.

(36) Il s'agit de la Lex Pompeia de provinciis de 52 av. J.-C. qui établit un intervalle de cinq ans entre une magistrature exercée à Rome et une promagistrature (en province). Ce texte fait l'objet d'interprétations diverses.

(37) À Narbonne, se tenait chaque année une assemblée de la province manifestant son loyalisme à l'empereur dans un sanctuaire de Rome et d'Auguste. Une inscription dont n'ont été conservés que cinq paragraphes montre que l'administration romaine avait la haute main sur cette assemblée. Les attributions du flamine impérial sont soigneusement fixées (Éric DELONG, Carte archéologique de la Gaule narbonnaise et le Narbonnais 11/1, Paris, C.I.D., 2002).

(38) Allusion au mariage avec l'archiduchesse Marie-Louise. 
d'histoire moderne et la lenteur regrettable avec laquelle les ouvrages demandés y parviennent.

Le jury a été amené, en tenant compte des circonstances et de la difficulté inégale des thèses, à donner la même note, $6{ }^{1 / 2}$ sur 10 aux trois candidats.

Les épreuves de géographie n'ont été que de force moyenne, les candidats, sauf peut-être l'un d'entre eux, n'ont pas témoigné d'un sens très net des choses géographiques. Faute de réfléchir suffisamment sur les limites et le but de cette science, ils ont affiché une importance démesurée et souvent exclusive aux notions auxiliaires que fournit par exemple la géologie. On a créé récemment le mot d'anthropogéographie. Ce terme n'est pas nécessaire, tout géographe doit songer constamment à l'homme quand il décrit la terre sinon il fait une vaine et inégale concurrence au géologue, au météorologiste, etc. et l'union des études d'histoire et de géographie devient inintelligible.

Les trois sujets proposés étaient les suivants :

$1^{\circ}$ ) Étudier les traits principaux de la géographie physique de l'Écosse.

$2^{\circ}$ ) État des connaissances sur la géographie physique et économique de l'Afrique australe.

$3^{\circ}$ ) Étudier une division de la Tunisie en régions naturelles.

M. Lefebvre a lu avec attention et intelligence les livres essentiels sur la géographie de l'Écosse et fait preuve de qualités d'ordre et de clarté. Mais l'esprit même de son étude laissait à désirer. Il s'est borné à décrire les trois régions écossaises, il ne s'est même appliqué sérieusement qu'aux questions d'orogénèse ; il n'a pas cherché à mettre en valeur l'influence des conditions physiques sur le développement historique et économique de l'Écosse. La note 6 lui a été donnée.

M. Thomas a beaucoup travaillé pour ordonner les renseignements nombreux qu'il avait recueillis sur l'Afrique australe. C'est pourquoi il a obtenu la note 7 . Il est à regretter que l'intérêt véritable de la question lui ait échappé. Il fallait étudier avant tout les régions aurifères et les étudier en géographe et en historien ; montrer que, comme celles de la Californie et de l'Australie, elles sont destinées, par l'afflux des émigrants et des capitaux, à devenir des pays d'exploitation agricole.

$M$. de $S^{t}$ Léger avec plus de pénétration a saisi les difficultés du troisième sujet. Pour expliquer les aspects variés de la nature tunisienne, il était nécessaire de tenir compte dans une juste mesure de facteurs très complexes. Le candidat y est à peu près parvenu, grâce à une maturité de 
jugement et à une souplesse d'esprit dignes d'éloges. Aussi a-t-il obtenu la note de $7 \frac{1}{2}$.

Les dernières épreuves ont mérité des notes plus élevées. M. de $S^{t}$ Léger qui avait étudié la vie de Clovis dans Grégoire de Tours ${ }^{39}$ a bien expliqué et critiqué le passage plus qu'à moitié légendaire où est raconté le mariage du roi des Francs avec Clotilde. Il a eu la note 9 sur 10. M. Lefebvre avait choisi un texte qui prête encore à de multiples discussions, le Modus tenendi Parliamentum Anglia ; il a commenté avec soin et avec plus de précision que dans ses précédentes épreuves, le chapitre sur l'Aide au roi ; on lui a accordé la note 8 . Enfin, M. Thomas a obtenu $7 \frac{1}{2}$ en critiquant le texte du traité d'Arras de 1414 donné par Monstrelet ${ }^{40}$. M. M. de $S^{t}$ Léger et Lefebvre ont déchiffré sur des fac-similés [sic] des textes latins des XII et XIII ${ }^{\mathrm{e}}$ siècles et ont mérité les notes 8 et $7 \frac{1}{2}$. L'explication d'une inscription grecque par M. Thomas lui a valu la note $6 \frac{1}{2}$.

L'ensemble des notes, qui a toujours dépassé la moyenne, a conduit le jury à déclarer les trois candidats dignes du diplôme. Convaincus par cette intéressante expérience de l'intérêt et de l'excellence de l'examen créé par l'arrêté du 28 juillet 1894, les professeurs d'histoire et de géographie de l'Université de Lille n'ont plus qu'à exprimer leur désir de voir cet examen devenir indépendant de l'agrégation, sans cesser d'en être un préambule nécessaire. Le jour qui semble assez prochain, où cette réforme sera accomplie, des modifications de détail pourront sans doute être faites par chaque université. Il est souhaitable par exemple que la question des coefficients des notes puisse être de nouveau examinée et résolue par les professeurs.

Signé : Ch. Petit-Dutaillis.

Philippe MARCHAND

IRHIS

Université de LILLE III Pmarchand356@numericable.fr

(39) Grégoire de TOURS, Histoire des Francs (Historia Francorum).

(40) Enguerran de Monstrelet, auteur d'une Chronique s'étendant de 1400 à 144 . Il est suspecté de s'y montrer favorable aux Bourguignons. 